\title{
The future of asthma research and development: a roadmap from the European Asthma Research and Innovation Partnership (EARIP)
}

\author{
Sarah Masefield ${ }^{1}$, Jessica Edwards ${ }^{2}$, Kjeld Hansen $^{3}$, Dominique Hamerlijnck ${ }^{3}$, \\ Karin Lisspers ${ }^{4}$, Marc van der Schee ${ }^{5}$, Liliana Silva ${ }^{6}, J^{\prime}$ ohn Matthews ${ }^{7}$, \\ Mina Gaga ${ }^{8}$, Ian Adcock ${ }^{9}$, Stephen Holgate ${ }^{10}$, Samantha Walker ${ }^{2}$ and \\ Pippa Powell ${ }^{1}$
}

Affiliations: ${ }^{1}$ European Lung Foundation, Sheffield, UK. ${ }^{2}$ Asthma UK, London, UK. ${ }^{3}$ European Lung Foundation. ${ }^{4}$ Dept of Public Health and Caring Science, Family Medicine and Preventive Medicine, Uppsala University, Uppsala, Sweden. ${ }^{5}$ Paediatric Respiratory Medicine, Academic Medical Centre University of Amsterdam, Amsterdam, The Netherlands. ${ }^{6}$ Community Care Unit, Matosinhos Local Health Unit, Matosinhos, Portugal. 'Genentech Inc., San Francisco, CA, USA. ${ }^{8} 7$ th Respiratory Medicine Dept, Athens Chest Hospital, Athens, Greece. ${ }^{9}$ Thoracic Medicine, Imperial College London, London, UK. ${ }^{10}$ Faculty of Medicine, Southampton General Hospital, Southampton, UK.

Correspondence: Pippa Powell, European Lung Foundation, 442 Glossop Road, Sheffield, S10 2PX, UK. E-mail: pippa.powellaeuropeanlung.org

@ERSpublications

Asthma outcomes will be transformed if the priorities in the EARIP research roadmap are addressed http://ow.ly/9maP307TVNk

Cite this article as: Masefield S, Edwards J, Hansen K, et al. The future of asthma research and development: a roadmap from the European Asthma Research and Innovation Partnership (EARIP). Eur Respir J 2017; 49: 1602295 [https://doi.org/10.1183/13993003.02295-2016].

\begin{abstract}
A coordinated European multi-stakeholder approach to asthma research
Asthma is highly prevalent and associated with high morbidity and mortality. It affects 30-50 million people in Europe, often starting in infancy and persisting throughout life. Asthma is a major global health challenge, affecting more than 300 million people worldwide and at least 10\% of all Europeans [1]. People with asthma live at risk of life-threatening asthma attacks, leading to over 500000 hospitalisations each year. Approximately $5-10 \%$ of asthma cases are so severe that current treatments do not work.

The Framework Programme 7-funded European Asthma Research and Innovation Partnership (EARIP; www.EARIP.eu) was established in 2013 to harmonise efforts to reduce mortality and morbidity from asthma by agreeing the most important research priorities across relevant stakeholders in Europe. This is essential to address the significant impact of asthma on the individual, healthcare systems and national and European economies, outlined in the accompanying editorial in this issue of the European Respiratory
\end{abstract}

Received: Nov 222016 | Accepted: Dec 052016

This article was endorsed by the ERS Science Council and Executive Committee in February 2017.

Support statement: This work was funded by the Horizon 2020 Framework Programme. The European Asthma Research and Innovation Partnership (EARIP) is funded under the European Union in the 7th Framework Programme (grant agreement 602077). Funding information for this article has been deposited with the Open Funder Registry.

Conflict of interest: S. Masefield and P. Powell are employees of the European Lung Foundation. D. Hamerlijnck and K. Hansen are patient representatives of the European Lung Foundation. Other disclosures can be found alongside this article at erj.ersjournals.com

Copyright OERS 2017 
Journal [2]. EARIP produced an evidence- and consensus-based list of the research priorities (a "roadmap") and investment needed to reduce asthma deaths and hospitalisations. By identifying the priorities, a coordinated effort can be made to fast-track change to better manage, prevent and cure asthma [3]. The roadmap will be the foundation on which future EU, national and international research funding programmes can transform asthma outcomes throughout Europe.

The roadmap is the product of a comprehensive multi-stage process, led by the European Lung Foundation and Asthma UK (figure 1) [4]. Multiple stakeholder groups informed the roadmap process to ensure scientific accuracy, clinical relevance and outcomes that reflect the priorities of patients and caregivers, with an overarching steering board. A final consensus workshop brought together 28 experts from 15 European countries comprising: patients, patient organisations, primary healthcare professionals (HCPs), secondary HCPs, researchers, industry representatives and policy influencers. A full list of the roadmap contributors is available at www.EARIP.eu/roadmap. The rest of this article describes its development and outcomes.

\section{Priorities for asthma research and development}

Following extensive literature review [5-19] and pan-European consultation [4], a final set of 15 research priorities were established (table 1).

Discussion surrounding the prioritisation exercise produced four overarching themes covering basic and clinical/applied research: 1) primary care and public health; 2) triggers and risk factors for asthma and exacerbations; 3) personalised medicine; and 4) self-management and adherence.

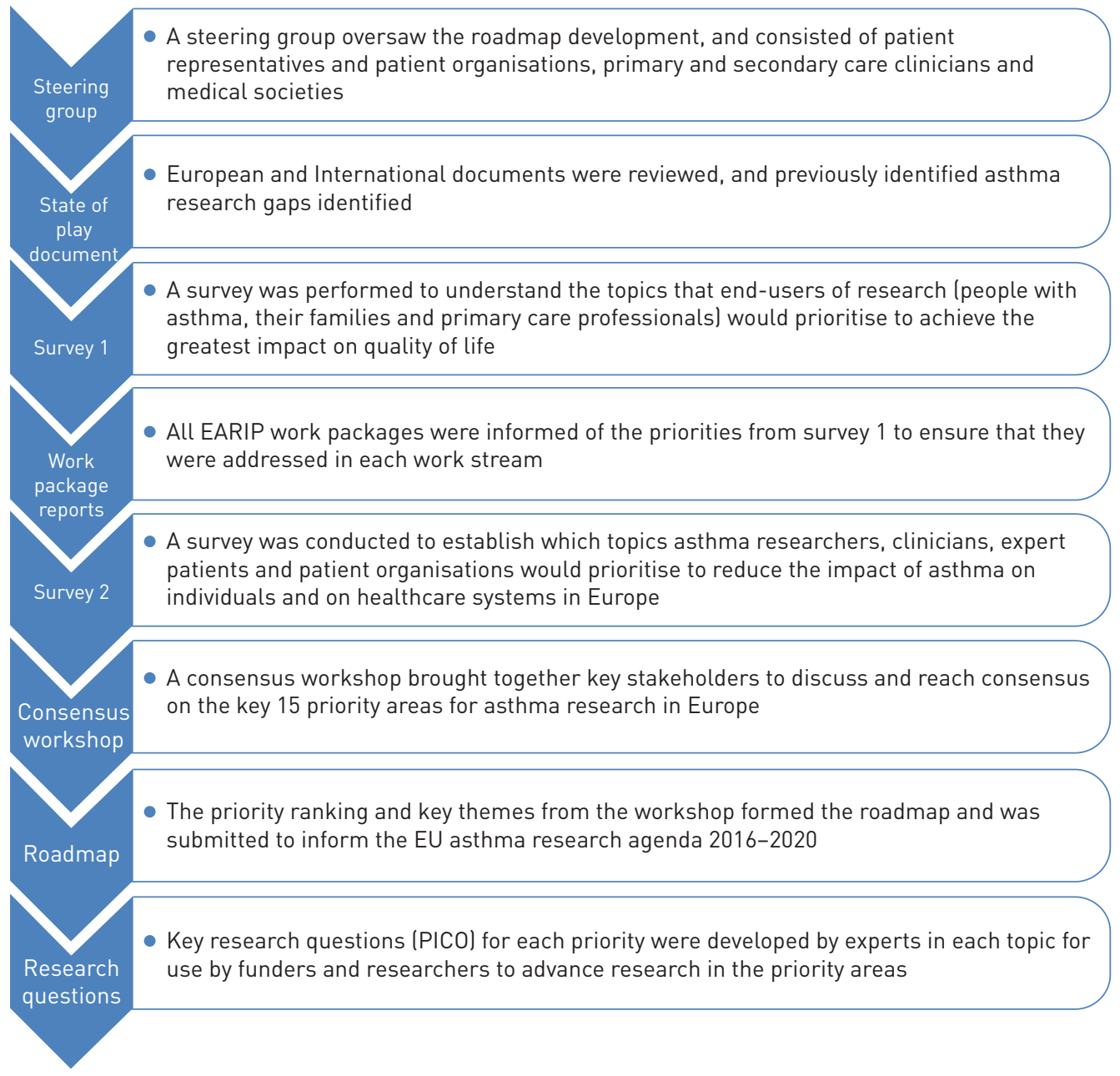

FIGURE 1 The roadmap development process. EARIP: European Asthma Research and Innovation Partnership; PICO: Population, Intervention, Comparator, Outcome. 
TABLE 1 The 15 priorities as ranked by the consensus workshop participants

\section{Rank Priority}

1 Identify, understand and better classify the different forms of asthma, their progression, and effect on airway inflammation and the immune system

2 Assess the effectiveness of patient-professional communication to develop patient-professional partnerships, for example to optimise self-management and adherence

3 Assess the effect of infections in early childhood, the long-term effects of anti-inflammatory treatments, and use of anti-viral drugs and vaccines

4 Assess impact, adoption and transferability of best practice in regional, national and European asthma programmes, care pathways and asthma clinics

5 Develop new treatments for the different types of asthma: treatment-resistant and steroid-resistant asthma, severe asthma, allergic asthma, hyperresponsive asthma

6 Develop tools for quick, accurate and low-cost diagnosis to distinguish asthma from other causes of breathlessness, cough and wheeze

7 Evaluate the implementation of supported self-management, the educational needs of patients and caregivers, and the challenges faced and training needs of professionals

8 Evaluate the role of lung function testing and new ways of measuring airway inflammation in monitoring asthma

9 Identify biomarkers for exacerbations and understand the interactions between biomarkers, risk and comorbidities

10 Understand the increase in asthma (both childhood asthma and different types of asthma, such as allergic and hyperresponsive asthmal to help develop primary and secondary prevention strategies

11 Assess the efficacy of existing and new drugs on different asthma phenotypes

12 Develop tools to assess asthma self-management and asthma inhaler technique in primary care settings

13 Explore the interaction between asthma, socioeconomic and psychological factors, and comorbidities to reduce the risk of severe exacerbations

14 Investigate the impact of environmental factors on asthma and exacerbations, such as air quality (indoor and outdoor), climate, allergens and microorganisms and UV radiation

15

Understand the impact of exposure to substances known to trigger asthma, and the impact of strategies that regulate and control this exposure

The 15 asthma research priorities as identified in the EARIP (European Asthma Research and Innovation Partnership) Roadmap process. The priorities are listed here in ranked order, with 1 representing the highest priority, as identified by consensus workshop participants. All 15 topics were agreed as high priority for future research.

\section{Primary care and public health}

Regional and national asthma programmes can be hugely valuable. However, there is currently only published literature available for programmes in Finland, France, Ireland, Italy, the Netherlands, Poland, Portugal and Turkey [20]. The successes and challenges of these programmes should be used as a basis for new regional and national programmes [21]. Alongside this, simple and quick tools are needed to assess the implementation of new programmes, especially in primary care settings.

Effective care pathways are an essential component of asthma programmes, with lung function testing and measuring airway inflammation key to ensuring accurate diagnosis and monitoring from childhood. In the consensus workshop, primary care professionals stressed the importance of developing an accurate, low-cost tool that can diagnose and give information on treatment effectiveness and adherence quickly in primary care settings. This tool would have application worldwide, especially in low-middle income countries [22, 23].

The disparity between lung function and the patient's experience (how they feel, if they notice any changes in their asthma, etc.) should be considered an assessment and monitoring need, and connecting patient-reported symptoms with their clinical condition presents an opportunity to develop a valuable clinical tool [21].

\section{Triggers and risk factors for asthma and exacerbations}

Environmental factors are a major problem in patients' daily lives, both in terms of quality of life and productivity; therefore, environmental factors, exacerbations and strategies to control exposure should be priority topics in both basic science and implementation research. Investigation is needed into the daily interactions between patients' asthma and their environment, and the role of environmental factors in infections and long-term asthma management in both adults and children. Specific factors identified were smoking, the impact of smoking on childhood development and pollution (especially air quality) [19]. 


\section{Personalised medicine}

Research into different asthma phenotypes and methods of stratifying and better understanding these phenotypes could have the greatest long-term impact of all the priorities. This should be coupled with companion diagnostics and an understanding of how different treatments can affect children in the long term, such as anti-inflammatory and anti-viral treatments and vaccines. Biomarker research is a vital component in the development of new treatments; in particular, identifying biomarkers for exacerbations, which would also enable greater understanding of the links between increased exacerbation risk and comorbidities [23-25].

Research and funding is needed to build on current work in this field, such as the big data project U-BIOPRED (2010-2015), which made major advances in our understanding of asthma by recruiting and characterising a large pan-European cohort of people with severe asthma $[15,16]$. Although advancements are being made in drug development, major challenges remain in securing funding for implementation studies, and bringing new drugs to market and being prescribed for patients [24]. Workshop participants highlighted the difficulties in progressing personalised medicine and making big clinical differences, such as adequate drug options for rarer and less-controlled types of asthma, and adequate delivery of inhaler treatment $[21,23]$. In order for this to happen the differences between asthmas and their phenotypes, and their impact on the airways and immune system must be fully understood.

Breakthroughs in asthma treatment are not easy and take a long time. They are also hampered by the lack of routine information sharing between primary and secondary care settings and pharmacological companies, with no requirement for clinicians to keep comparable records of a drug's performance in their patients. Current and new drugs in circulation should be evaluated for their efficacy within real-world clinical practice, with a wide range of patients over time. There should also be more data sharing between studies, with potential for structures such as the European Respiratory Society Research Agency to do this.

\section{Self-management and adherence}

The role of primary care professionals in promoting self-management brings a need for specific training on how to empower and motivate self-management in patients, especially in communicating the effectiveness of existing drugs for the majority of people with asthma when taken correctly [21]. Research would help HCPs better understand how to assess a patient's level of health literacy, engage patients with variable levels of interest in self-management, and adapt oral and written communications to meet each patient's needs and preferences. Other key areas for investment are research into the differences in access to healthcare services and treatment, and the impact of asthma on different patient populations [23].

Advances and breakthroughs in new drugs and supported self-management must be underpinned by real-world evaluation with people with different types of asthma and, importantly, with patients with a range of adherence issues (behavioural, complex drug regimes, age-related issues, poor inhaler technique, etc.) $[20,23,25]$. Asthma-specific self-management research is essential to better understand this, as generic non-condition specific research risks overlooking the influence of aspects such as medicine regimes, inhaler technique and triggers for exacerbations [18].

New technologies have an important role to play in primary prevention; research is needed to develop new and effective public awareness initiatives on asthma and air quality (indoor and outdoor), smoking and allergies [13]. For example, further innovations in mobile and electronic health platforms have the potential to revolutionise self-management by providing support and enabling professionals to assess/ monitor their patient's self-management remotely [21].

\section{Making the priorities a research reality}

Within each of the 15 priorities, specific research questions using the PICO (Population, Intervention, Comparator, Outcome) approach have been developed by experts in each field to add further specificity and direction, making it easier for funders and research commissioners, including the European Commission, research institutes and industry, to adopt them as part of their research work plans (supplementary material).

\section{Summary}

A unified approach to innovation is needed to address the challenge of asthma in Europe. It is the opinion of the EARIP consortium and associated members (comprising most asthma networks, societies and professional groups) that if all of these research priority areas were funded and the 15 research questions addressed, asthma outcomes would be transformed and avoidable use of healthcare systems eradicated, resulting in significant financial savings.

The realisation of this vision through coordinated efforts at a European level is the only way to achieve the change needed to reduce asthma deaths and hospitalisations and improve productivity and quality of life 
for people with asthma. Integral to this is maintaining and growing the networks established through EARIP professional societies, patient organisations and EU-funded programmes.

\section{Acknowledgements}

Thanks go to all EARIP partners: Sebastian Johnston, Sven-Erik Dahlen, Giuseppe De Carlo, Susanna Palkonen, Luis Garcia-Marcos, Nikos Papadopoulos, Aggeliki Androutsopoulou, Cezmi Akdis, Maciek Kupczyk, John Riley, David Myles, Mark Levick, Ratko Djukanovic, Piotr Kuna; and to the remaining workshop participants and PICO question developers: Guy Joos, Hilary Pinnock, Antonius Schneider, Liliana Silva, Jacky Smith, Otto Spranger, Peter Sterk, Phil Taverner, Ian Adcock, Peter Barnes, Elisabeth Bel, Breda Flood, Liliya Gentet, Christina Gratziou, Ian Hall, Rob Horne, Katherine Cowan, Leanne Metcalf, Erika Kennington, Courtney Coleman, Liam Heaney, Hector Ortega, Samantha Sonnappa, Rik Loijmans, Ildiko Horvath, John Riley, Sarah Kirby, Lucy Yardley, Ben Ainsworth, Paul Cullinan, Angela Simpson. The final thanks go to the EARIP Patient Advisory Group and all who completed the online surveys.

\section{References}

1 Gibson J, Loddenkemper R, Sibille Y, et al., eds. The European Lung White Book. 2nd Edn. Sheffield, European Respiratory Society, 2013.

2 Papadopoulos NG, Androutsopoulou A, Akdis C, et al. Asthma research in Europe: a transformative agenda for innovation and competitiveness. Eur Respir J 2017; 49: 1602294.

3 Masefield S, Powell P, Kennington EJ, et al. Driving investment in asthma research in Europe: priorities to prevent, cure and manage asthma more effectively. Am J Respir Crit Care Med 193; 2016: A2668.

4 Masefield S, Metcalf L, Powell $\mathrm{P}$, et al. What is important to people with asthma: maximising research impact? Eur Respir J 2015; 46; Suppl 59, OA4479.

5 Chung KF, Wenzel SE, Brozek JL, et al. International ERS/ATS guidelines on definition, evaluation and treatment of severe asthma. Eur Respir J 2014; 43; 343-373.

6 Carlsen K-H, Gerritsen J, eds. Paediatric asthma (ERS Monograph). Sheffield, European Respiratory Society, 2012.

7 European Respiratory Roadmap: Recommendations for the future of respiratory medicine. Sheffield, European Respiratory Society, 2011.

8 Demoly P, Tanno LK, Akdis CA, et al. Global classification and coding of hypersensitivity diseases - An EAACI WAO survey, strategic paper and review. Allergy 2014; 69: 559-570.

9 Custovic A, Johnston SL, Pavord I, et al. EACCI position statement on asthma exacerbations and severe asthma. Allergy 2013; 68: 1520-1531.

10 European Academy of Allergy and Clinical Immunology. Global Atlas of Asthma. Available at: www.eaaci.org/ attachments/Global\%20Atlas\%20of\%20Asthma.pdf Date last accessed: March 1, 2015. Date last updated: 2013.

11 Global Asthma Network. The Global Asthma Report. Available at: www.globalasthmareport.org/resources/Global_ Asthma_Report_2014.pdf Date last accessed: January 27, 2015. Date last updated: 2014.

12 Institute for Healthcare Communication. Impact of communication in healthcare. Available at: http:// healthcarecomm.org/about-us/impact-of-communication-in-healthcare Date last accessed: July 25, 2016. Date last updated: July 2011.

13 Papadopoulos N, Agache I, Bavbek S, et al. Research needs in allergy: an EACCI position paper, in collaboration with EFA. Clinical Transl Allergy 2012; 2: 21.

14 Pinnock $\mathrm{H}, \varnothing$ stremb A, Rodríguez $\mathrm{MR}$, et al. Prioritising the respiratory research needs of primary care: the International Primary Care Respiratory Group (IPCRG) e-Delphi exercise. Prim Care Respir J 2012; 21: 19-27.

15 Fleming L, Murray C, Bansal AT, et al. The burden of severe asthma in childhood and adolescence: results from the paediatric U-BIOPRED cohorts. Eur Respir J 2015; 46: 1322-1333.

16 Shaw DE, Sousa AR, Fowler SJ, et al. Clinical and inflammatory characteristics of the European U-BIOPRED adult severe asthma cohort. Eur Respir J 2015; 46: 1308-1321.

17 Ring J, Akdis C, Behrendt H, et al. Davos declaration: allergy as a global problem. Allergy 2012; 67: 141-143.

18 Sims EJ, Price D, Haughney J, et al. Current control and future risk in asthma management. Allergy Asthma Immunol Res 2011; 3: 217-225.

19 Georas SN, van Wijngaarden E, Rich DQ. Air pollution and asthma incidence: doubt no more? Lancet Respir Med 2015; 3: 902-903.

20 Selroos O, Kupczyk M, Kuna P, et al. National and regional asthma programmes in Europe. Eur Respir Rev 2015 24: $474-483$.

21 De Carlo G, De Vocht J, Edwards JL, et al. Transforming asthma outcomes through co-ordinated national and regional health initiatives: review and policy recommendations. Am J Respir Crit Care Med 2016; 193: A2670.

22 Garcia-Marcos L, Edwards JL, Kennington EJ, et al. What are the priorities for future research into diagnostic tools for asthma? A Pan-EU consensus exercise. Am J Respir Crit Care Med 2016; 193: A1430.

23 Garcia-Marcos L, Edwards JL, Kennington EJ, et al. What asthma self-management tools and systems should have higher priority in future research: a Delphi exercise from the EU. Am J Respir Crit Care Med 2016; 193: A2669.

24 Djukanovic R, Varkonyi-Sepp J, Varkonyi B, et al. What is needed to speed up the development of new asthma treatments in Europe: a pan-European perspective. Am J Respir Crit Care Med 2016; 193: A4962.

25 Johnston S, Edwards MR, Schwarze J, et al. Where next in asthma research? A review of current understanding and future focus to prevent and cure asthma. Am J Respir Crit Care Med 2016; 193: A2923. 